\title{
A Novel High Sensitivity Refractive Index Sensor Based on Multi-Core Micro/Nano Fiber
}

\author{
Yuefeng $\mathrm{QI}^{1,2}$, Jin ZHANG ${ }^{1 *}$, Qi FENG ${ }^{1}$, Xin ZHANG ${ }^{1}$, \\ Yanyan LIU ${ }^{1,3}$, and Ying HAN ${ }^{1,2}$ \\ ${ }^{1}$ School of Information Science and Engineering, Yanshan University, Qinhuangdao 066004, China \\ ${ }^{2}$ Key Laboratory for Special Fiber and Fiber Sensor of Hebei Province, Qinhuangdao 066004, China \\ ${ }^{3}$ BaYin GuoLeng Vocational and Technical College, Korla 841000, China \\ ${ }^{*}$ Corresponding author: Jin ZHANGＩE-mail: 774840864@qq.com
}

\begin{abstract}
A refractive index sensor based on a multi-core micro/nano fiber is proposed for low refractive index solutions. At first, the mode field distribution of the tapered multi-core fiber is analyzed with the finite element model (FEM). After that, the relationship between the refractive index sensitivity and the diameter of the multi-core micro/nano fiber is calculated. At last, four sensors with different sizes are explored, and when the taper length is $16.20 \mathrm{~mm}$, the refractive index sensitivity of the sensor can reach $5815.50 \mathrm{~nm} / \mathrm{RIU}$, which agrees with the theoretical analysis. The refractive index measurement error is less than $0.5 \%$, which has a high practical application value. The longer the taper length is, the smaller the fiber diameter is. According to the theoretical analysis, when the fiber diameter is less than $4.864 \mu \mathrm{m}$, the structure sensor's refractive index sensitivity is higher than $10000 \mathrm{~nm} / \mathrm{RIU}$. At the same time, when the sensor's taper length is $15.99 \mathrm{~mm}$, its temperature sensitivity is $-0.1084 \mathrm{~nm} /{ }^{\circ} \mathrm{C}$. Compared with single-mode fiber, the sensor proposed here has the advantages of stability, compact structure, and high sensitivity, which has a potential in the field of seawater salinity measurement.
\end{abstract}

Keywords: Fiber optic sensor; refractive index measurement; multi-core fiber

Citation: Yuefeng QI, Jin ZHANG, Qi FENG, Xin ZHANG, Yanyan LIU, and Ying HAN, “A Novel High Sensitivity Refractive Index Sensor Based on Multi-Core Micro/Nano Fiber," Photonic Sensors, 2019, 9(3): 197-204.

\section{Introduction}

The measurement of refractive index plays an important role in the fields of biological, chemical, and water environmental monitoring [1]. The minimum deflection angle method [2] and the Abbe refractometer instrument [3] are two main techniques in the measurement of liquid refractive index. These technologies are low in sensitivity and require a lot of manual operations, which are not suitable for practical industrial applications. In recent years, the fiber optic sensor has attracted extensive attention in the field of refractive index measurement, due to its simple structure, anti-electromagnetic interference, corrosion resistance, and high sensitivity [4].

The fiber refractive index sensor mainly includes fiber grating refractive index sensors [5] and optical fiber interferometric refractive index sensors [6]. In 2017, Shen et al. produced a long-period fiber grating refractive index sensor, with a period of $25 \mu \mathrm{m}$ ultraviolet (UV) inscribed and characterized.

Received: 28 November 2018 / Revised: 10 April 2019

(C) The Author(s) 2019. This article is published with open access at Springerlink.com

DOI: $10.1007 / \mathrm{s} 13320-019-0554-9$

Article type: Regular 
The sensor's refractive index sensitivity is $312.5 \mathrm{~nm} / \mathrm{RIU}$ in the range of 1.315 to 1.395 [7]. However, fiber grating refractive index sensors are costly to manufacture and cannot meet the requirements of ultra-high sensitivity measurement, which limits their further application in the field of refractive index measurement. Compared with the fiber grating refractive index sensor, the fiber interferometric sensor has the advantages of stability, low production cost, and high sensitivity [8]. In 2017, Cheng et al. produced a Fabry-Perot interferometric refractive index sensor. In this paper, a refractive index-modified dot (RIMD) near the end face of a single mode fiber is induced by femtosecond laser. Then, a Fabry-Perot interferometer is formed by the RIMD and fiber end face. This sensor has an ultra-high sensitivity of $2523.2 \mathrm{~dB} / \mathrm{RIU}$ at RI of 1.435 [9]. However, the aforementioned fiber interferometric sensor has a low sensitivity in the low refractive index range of $1.33-1.34$.

In this paper, a multi-core micro/nano fiber [10] interferometric sensor for low refractive index sensing has been proposed. The sensor consists of single mode fiber (SMF), 105/125 $\mu$ m multi-mode fiber (MMF), and three-core fiber (TCF). Theoretical simulations show that if the fiber diameter is smaller than $4.864 \mu \mathrm{m}$, the refractive index sensitivity of the sensor can reach as high as $10000 \mathrm{~nm} /$ RIU. Then, four different sensors with taper lengths of $14.50 \mathrm{~mm}, 15.99 \mathrm{~mm}, 16.10 \mathrm{~mm}$, and $16.20 \mathrm{~mm}$ are fabricated. Experimental results show that the refractive index sensitivities of $1920.79 \mathrm{~nm} / \mathrm{RIU}, \quad 2665.06 \mathrm{~nm} / \mathrm{RIU}$, $3509.44 \mathrm{~nm} / \mathrm{RIU}$, and $5815.50 \mathrm{~nm} / \mathrm{RIU}$ are achieved, respectively. For the sensor with $16.20 \mathrm{~mm}$ taper length, the measurement error is less than $0.5 \%$ in the range of 1.332-1.34. At the end of the paper, the temperature sensing experiments are also carried out by this multi-core micro/nano fiber interferometric sensor. When the taper length is $15.99 \mathrm{~mm}$, the temperature sensitivity of $-0.1084 \mathrm{~nm} /{ }^{\circ} \mathrm{C}$ is obtained.

\section{Theoretical analysis}

The structure of multi-core micro/nano fiber sensor is shown in Fig. 1. After the fiber is tapered, the lights transmit in the eccentric, and the intermediate cores of the TCF have optical path difference, which will result in interferences. Since the diameter of the cone is small, the core's effective refractive index of the TCF is affected by the external solution refractive index, and the effective refractive index of the eccentric core is more affected by the external solution than that of the intermediate core.

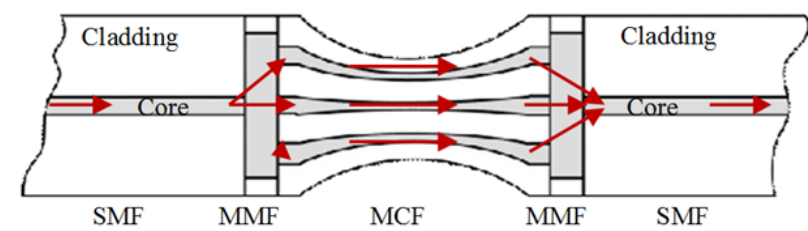

Fig. 1 Multi-core micro/nano fiber interferometric sensor structure.

The TCF model is constructed for numerical simulation, and its finite element model is shown in Fig. 2. The TCF's transmission modes are calculated by FEM, then the effective refractive index value corresponding to different modes are obtained. When the external refractive index is 1.3320 , the simulation results of the effective refractive index and propagation constant of the TCF is shown in Fig. 3. From Fig. 3, the effective refractive index and the propagation constant of $\mathrm{HE}_{11}$ mode become larger as the diameter increases.

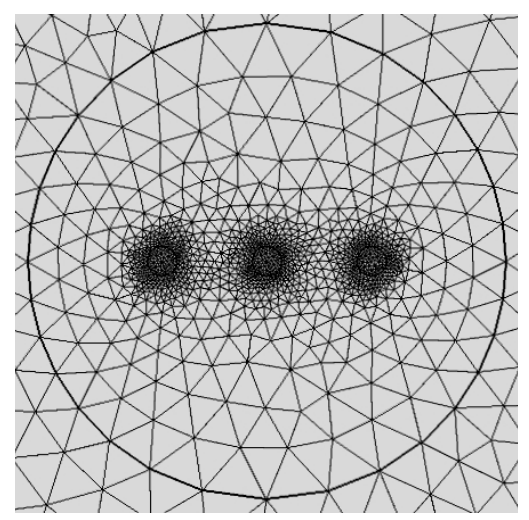

Fig. 2 Finite element simulation. 


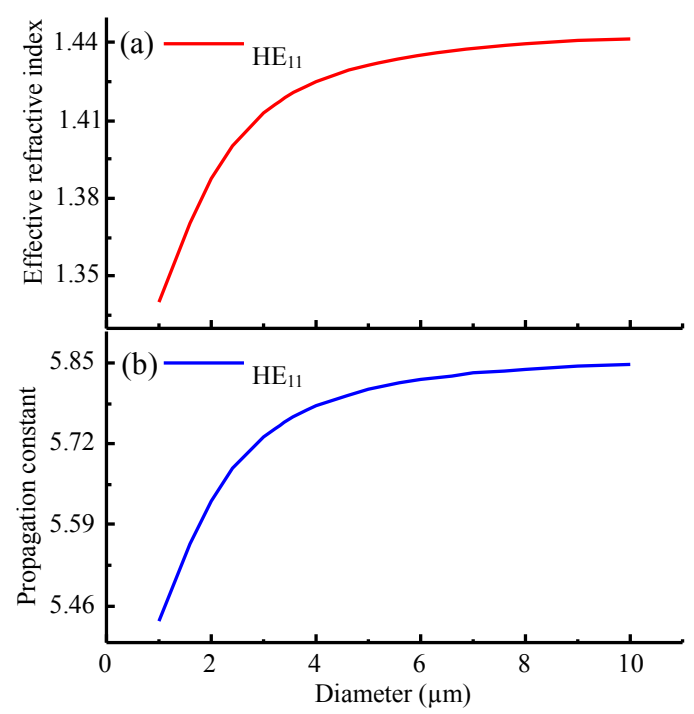

Fig. 3 Numerical results: (a) effective refractive index and (b) propagation constant.

Multi-core micro/nano fiber combines mode interference and strong evanescent field characteristics, which can monitor the change of external refractive index by using the drift of interference spectrum. The expression of the refractive index sensitivity $S_{\mathrm{RI}}[11]$ is

$$
S_{\mathrm{RI}}=\frac{d \lambda}{d n_{\mathrm{SRI}}}=\frac{\lambda\left[\partial\left(\Delta n_{\mathrm{eff}}\right) / \partial n_{\mathrm{SRI}}\right]}{\Delta n_{\mathrm{eff}}-\lambda \partial\left(\Delta n_{\mathrm{eff}}\right) / \partial \lambda}=\frac{\lambda}{G} \frac{\partial\left(\Delta n_{\mathrm{eff}}\right)}{\partial n_{\mathrm{SRI}}}(1)
$$

where $\lambda$ is the wavelength of the incident light, $\Delta n_{\text {eff }}$ is the effective refractive index difference between the intermediate and the eccentric core of the TCF, $n_{\mathrm{SRI}}$ is the refractive index value of external liquid, and $G$ is the group effective refractive index difference. In Fig.4, the relationship between the refractive index sensitivity $S_{\mathrm{RI}}$ and the diameter of the multi-core micro/nano fiber is shown when $n_{\mathrm{SRI}}=1.3320$.

It can be seen from Fig. 4 that there is a turning point at the diameter of $3.7 \mu \mathrm{m}$. The $S_{\mathrm{RI}}$ curve has opposite values on each side of the turning point. Near the turning point, the value of refractive index sensitivity is extremely high. The $10^{4} \mathrm{~nm} / \mathrm{RIU}$ ultra-refractive index sensitivity is readily achieved when the diameter of the fiber is tapered to be close to the turning point. However, there is an interference cut-off region at the left side of the turning point. In addition, the smaller the diameter is, the more fragile the fiber is. So the micro-fiber diameter should be controlled to the right side of the turning point.

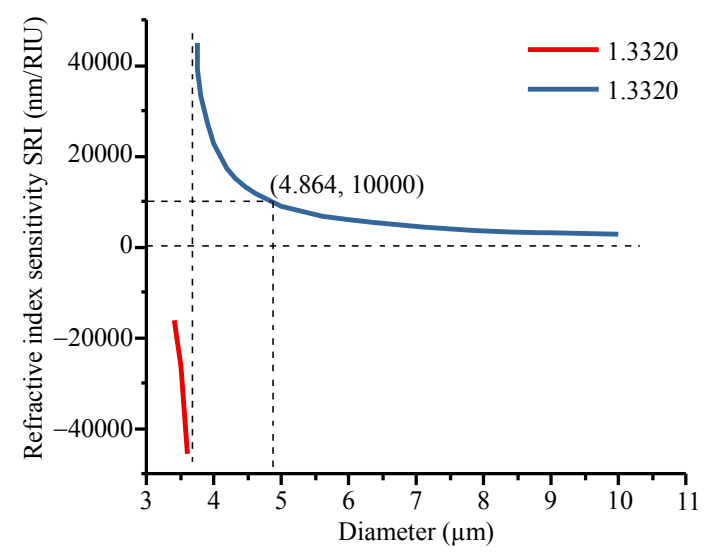

Fig. 4 Refractive index sensitivity when the external refractive index is 1.3320 .

\section{Experiment and result analysis}

\subsection{Experimental system construction}

The structure of the multi-core micro/nano fiber sensor proposed here consists of the SMF-MMF-TCF-MMF-SMF scheme which is shown in Fig. 5. Both ends of the TCF are fused with two sections of MMFs of $1 \mathrm{~mm}$ length, then the other ends of the two MMFs are fused with two SMFs, separately. The cross-section of MMF, TCF, and the electron microscope picture of the TCF are shown in Fig. 6. The MMF used in the sensor has a core diameter of $105 \mu \mathrm{m}$. The three cores of the TCF are arranged in a line with core diameter of $8 \mu \mathrm{m}$ and core to core spacing of $28 \mu \mathrm{m}$.

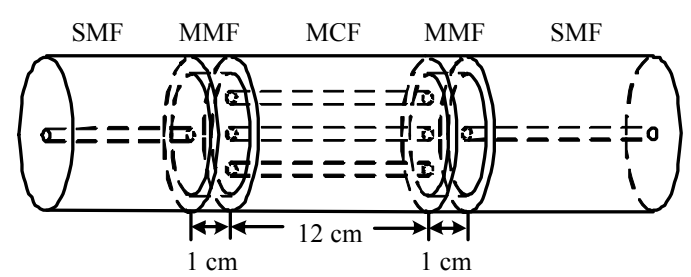

Fig. 5 SMF-MMF-MCF-MMF-SMF structure.

The fiber fusion splicer model is S178A of Furukawa Electric. SM-MM automatic welding mode is used to the fusion of SMF and MMF, and manual 
welding mode is employed in the fusing between MMF and MCF. The fused structure is shown in Fig. 7. After the welding is completed, the multi-core fiber is slowly stretched to make the taper angle smooth, so as to reduce the interference phenomenon of different propagation modes caused by the taper. The smooth taper angle can be achieved by slowing down the taper speed and adjusting the tightness of the clamp.

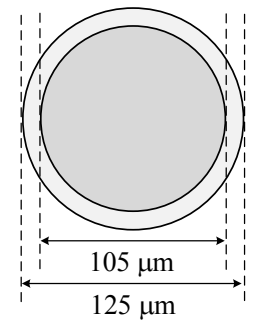

(a)

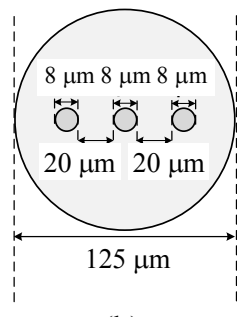

(b)

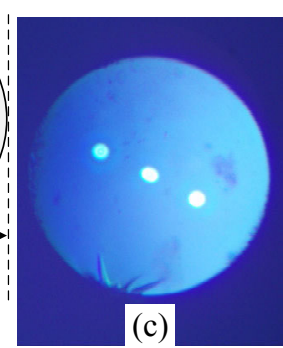

(c)
Fig. 6 Fiber cross section: (a) the 105/125 $\mu \mathrm{m}$ MMF cross-section, (b) the TCF cross-section, and (c) the TCF electron microscope observation.

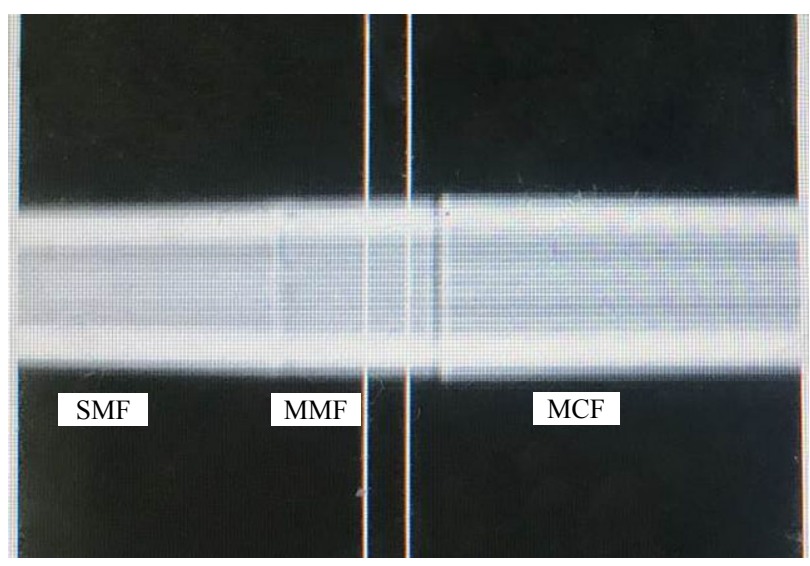

Fig. 7 Fused structure.

By this method, four different sensors with taper lengths of $14.50 \mathrm{~mm}, 15.99 \mathrm{~mm}, 16.10 \mathrm{~mm}$, and $16.20 \mathrm{~mm}$ are fabricated, corresponding to the waist cone diameters of $10 \mu \mathrm{m}, 8.56 \mu \mathrm{m}, 7.45 \mu \mathrm{m}$, and $5.92 \mu \mathrm{m}$, respectively.

\subsection{Refractive index sensing experiment}

The refractive index sensing setup is shown in Fig. 8. The tapered area is fixed on a piece of glass to ensure that the sensing area is in the straight state. Then, the whole sensor is immersed in the $\mathrm{NaCl}$ solution.

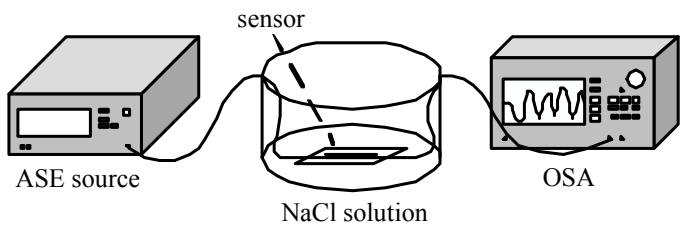

Fig. 8 Refractive index sensing experimental system.

The interference spectra of the four sensors with different taper lengths are shown in Fig. 9. As the length of the taper increases, the free spectral width of the interference spectrum becomes narrower.

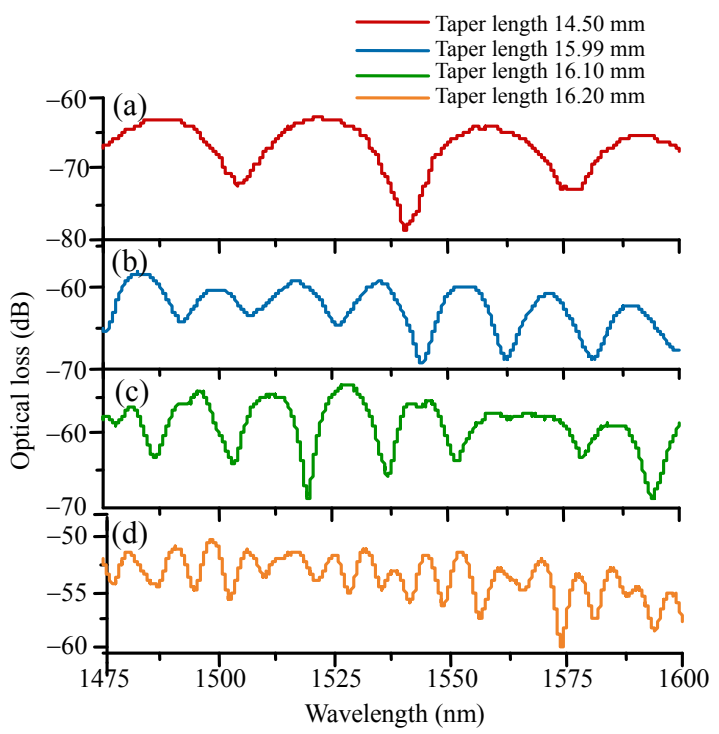

Fig. 9 Spectrogram of sensors with different taper lengths: (a) sensor with a taper length of $14.50 \mathrm{~mm}$, (b) sensor with a taper length of $15.99 \mathrm{~mm}$, (c) sensor with a taper length of $16.10 \mathrm{~mm}$, and (d) sensor with a taper length of $16.20 \mathrm{~mm}$.

Spectra and the relationship curves between the wavelength and refractive index for different taper lengths are shown in Fig. 10. It can be seen from Figs. 10(a), 10(c), 10(e), and 10(g) that as the refractive index of the external solution increases, the transmission spectrum is red-shifted. This is because that as the refractive index increases, the optical path difference between the intermediate core and the eccentric core changes. According to the simulation results of Fig. 4, when the diameter of the TCF is larger than $3.7 \mu \mathrm{m}$, the refractive index sensitivity has a positive value, which results in the red shift of the spectrum. The experimental data are coincided with the simulation results.

By analyzing the relationship between the trough drifts and the refractive index change of $\mathrm{NaCl}$ 
solution, the refractive index sensitivity curves of four sensors are obtained [Figs. 10(b), 10(d), 10(f), and 10(h)]. From Figs. 10(b), 10(d), 10(f), and 10(h), the sensor proposed here is very sensitive and linear to the refractive index change. When the taper length is $14.50 \mathrm{~mm}, 15.99 \mathrm{~mm}, 16.10 \mathrm{~mm}$, and $16.20 \mathrm{~mm}$, the SRI can reach as high as $1920.79 \mathrm{~nm} / \mathrm{RIU}, 2665.06 \mathrm{~nm} / \mathrm{RIU}, 3509.44 \mathrm{~nm} / \mathrm{RIU}$, and $5815.50 \mathrm{~nm} / \mathrm{RIU}$, respectively.
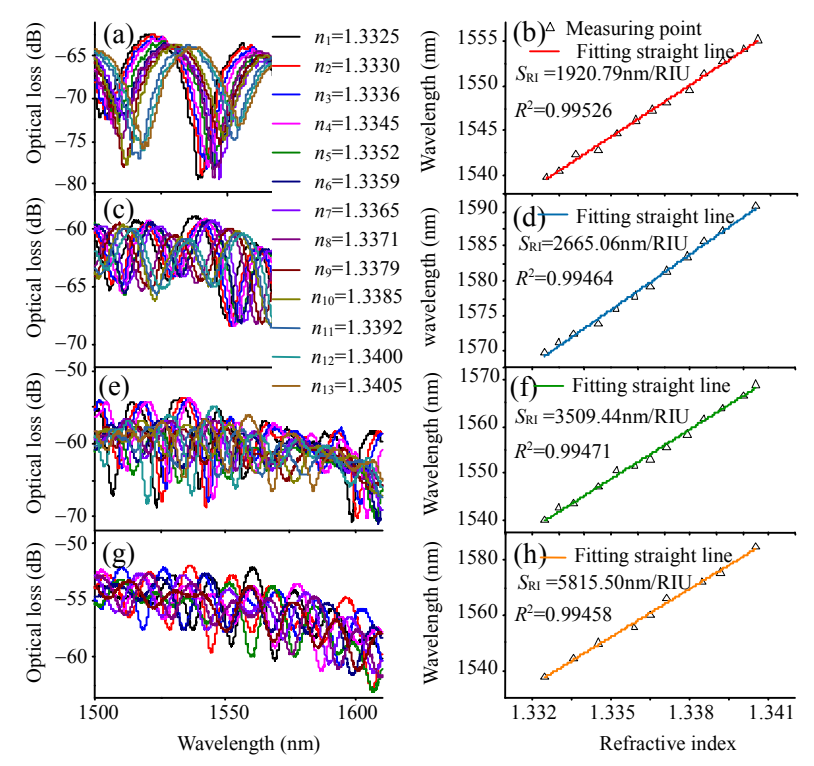

Fig. 10 Spectra and the relationship curves between wavelength and refractive index for different taper lengths: (a) spectrogram of the taper $14.5 \mathrm{~mm}$, (b) relationship curve of the taper $14.5 \mathrm{~mm}$, (c) spectrogram of the taper $15.99 \mathrm{~mm}$, (d) relationship curve of the taper $15.99 \mathrm{~mm}$, (e) spectrogram of the taper $16.1 \mathrm{~mm}$, (f) relationship curve of the taper $16.1 \mathrm{~mm}$, (g) spectrogram of the taper $16.2 \mathrm{~mm}$, and (h) relationship curve of the taper $16.2 \mathrm{~mm}$.

As mentioned in Section 3.1, when the taper lengths are $14.50 \mathrm{~mm}, 15.99 \mathrm{~mm}, 16.10 \mathrm{~mm}$, and $16.20 \mathrm{~mm}$, the corresponding waist cone diameters are $10.00 \mu \mathrm{m}, 8.56 \mu \mathrm{m}, 7.45 \mu \mathrm{m}$, and $5.92 \mu \mathrm{m}$, respectively. Table 1 shows the differences between the numerical simulation and the actual measurement results. It can be seen from Table 1 that the simulation and experimental results show the same regularity, as the length of the taper increases, the refractive index sensitivity becomes larger. However, the $S_{\mathrm{RI}}$ value obtained by the experiment is always smaller than that obtained by the theoretical simulation. The main reason is that the tapered region is approximately equivalent to a trapezoid when building a simulation model, while the actual tapered region is gradually changed.

Table 1 Comparison of simulation results and experimental results under different taper lengths.

\begin{tabular}{ccc}
\hline $\begin{array}{c}\text { Taper length } \\
(\mathrm{mm})\end{array}$ & $\begin{array}{c}\text { Simulated refractive } \\
\text { index sensitivity } \\
(\mathrm{nm} / \mathrm{RIU})\end{array}$ & $\begin{array}{c}\text { Experimental refractive } \\
\text { index sensitivity } \\
(\mathrm{nm} / \mathrm{RIU})\end{array}$ \\
\hline 14.50 & 2643.06 & 1920.79 \\
15.99 & 3290.05 & 2665.06 \\
16.10 & 4011.40 & 3509.44 \\
16.20 & 6126.31 & 5815.50 \\
\hline
\end{tabular}

The comparison results of sensor with other types of refractive index sensors are shown in Table 2. It can be seen from Table 2 that the modal interference sensor based on the multi-core micro/nano fiber has a higher refractive index sensitivity.

Table 2 Sensor refractive index sensitivity using different types of fibers.

\begin{tabular}{cc}
\hline Fiber type & $\begin{array}{c}\text { Refractive index sensitivity } \\
\text { (nm/RIU) }\end{array}$ \\
\hline Three-core fiber & 5815.50 \\
Three-clad fiber [12] & 528.86 \\
Fine core fiber [13] & 205.42 \\
Two-core photonic crystal fibers [14] & 198.77 \\
Gradient multimode fiber [15] & 152.24 \\
\hline
\end{tabular}

\subsection{Accuracy detection of refractive index sensor}

Firstly, two standard solutions are prepared, and their refractive index values are 1.3371 and 1.3385 measured by Abbe refractometer. Then, six seawater samples are sampled at three different locations and two different depths of the sea. They are marked as A shallow, A deep, B shallow, B deep, C shallow, C deep.

The refractive indexes of the six seawater samples are measured by the sensor with $16.20 \mathrm{~mm}$ taper length. The spectrum of the solution under tested and the standard liquid are shown in Fig. 11. The spectra of A shallow, B shallow, and B deep are compared with the spectrum of standard liquid $n=1.3371$. The spectra of A deep, $\mathrm{C}$ shallow and C deep are compared with the spectrum of standard liquid $n=1.3385$. 

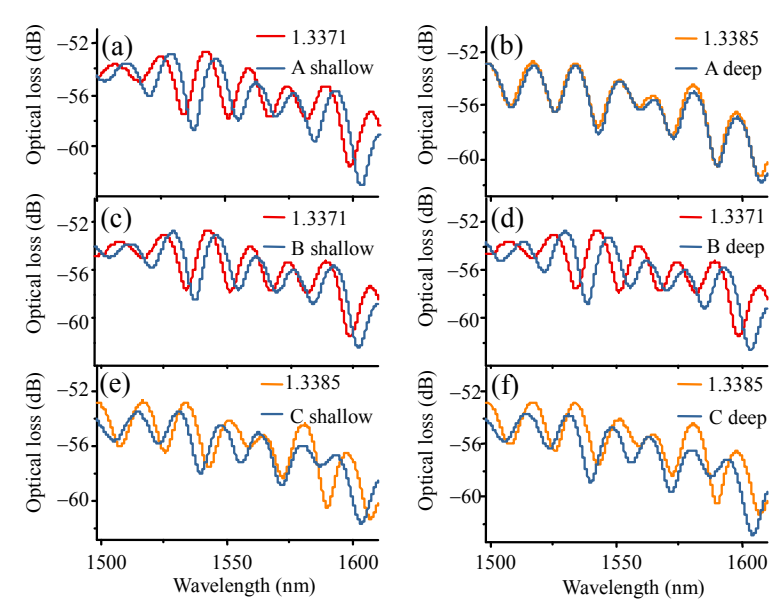

Fig. 11 Comparison of seawater samples with the standard spectrum: (a) comparison of "A shallow" sample and $n=1.3371$,

(b) comparison of "A deep" sample and $n=1.3385$,

(c) comparison of "B shallow" sample and $n=1.3371$,

(d) comparison of "B deep" sample and $n=1.3371$,

(e) comparison of " $\mathrm{C}$ shallow" sample and $n=1.3385$, and

(f) comparison of " $\mathrm{C}$ deep" sample and $n=1.3385$.

Table 3 Error analysis of measurement value and standard value of different seawater samples.

\begin{tabular}{cccc}
\hline Seawater sample & $\begin{array}{c}\text { Sensor } \\
\text { measurement }\end{array}$ & Standard value & $\begin{array}{c}\text { Sensor } \\
\text { measurement error } \\
(\%)\end{array}$ \\
\hline A shallow & 1.338065 & 1.3380 & 0.0486 \\
A deep & 1.338589 & 1.3385 & 0.0665 \\
A shallow & 1.338028 & 1.3379 & 0.0957 \\
A deep & 1.338122 & 1.3380 & 0.0912 \\
A shallow & 1.338268 & 1.3378 & 0.3498 \\
A deep & 1.338402 & 1.3380 & 0.3005 \\
\hline
\end{tabular}

According to the data above, the refractive index value of the unknown seawater sample can be calculated. For accuracy, more than one trough is selected as reference point, several refractive indexes of the tested samples are obtained, and their average values are computed as the final result. Meanwhile, the refractive indexes of the seawater samples are also measured by an Abbe refractometer as standard values. The error analysis of the measured values is shown in Table 3 . It can be seen from Table 3 that the measurement error of the structure sensor described in this paper is below $0.5 \%$.

\subsection{Temperature sensing experiment}

The temperature experimental setup is shown in Fig. 12. The ambient temperature of the sensor can be controlled by the temperature control box.

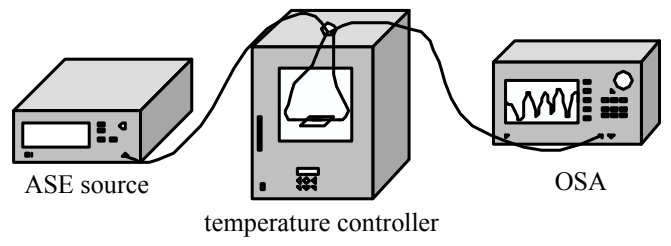

Fig. 12 Temperature experimental setup.

The sensor with $15.99 \mathrm{~mm}$ taper length is used to perform the temperature sensing experiments. The transmission spectra at different temperatures are measured and analyzed in Fig. 13. As the temperature increases, the spectrum shifts to the short-wave direction, as shown in Fig. 13(a), and the temperature sensitivity of the sensor is $-0.1084 \mathrm{~nm} /{ }^{\circ} \mathrm{C}$ with a good linearity, as shown in Fig. 13(b).

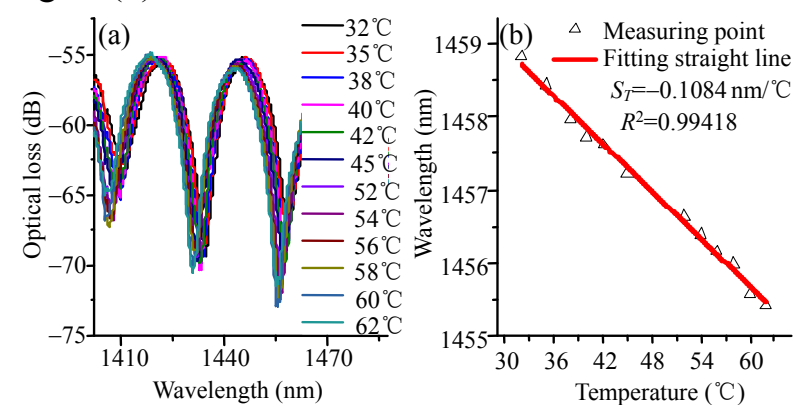

Fig. 13 Spectra and temperature characteristics: (a) spectra at different temperatures and (b) wavelength and temperature relationship curve.

From [16], the sensor can also be cascaded with the fiber Bragg grating (FBG) [17] to eliminate the cross-sensitivity of refractive index and temperature by

$$
\left[\begin{array}{l}
\Delta \lambda_{m 1} \\
\Delta \lambda_{m 2}
\end{array}\right]=\left[\begin{array}{ll}
A & B \\
C & D
\end{array}\right]\left[\begin{array}{c}
\Delta n \\
\Delta T
\end{array}\right]
$$

where $\Delta \lambda_{m 1}$ and $\Delta \lambda_{m 2}$ are the wavelength drifts between the FBG center wavelength and the observation point near $1550 \mathrm{~nm} ; A$ and $B$ are the FBG refractive index and temperature sensitivity, respectively; $C$ and $D$ are the refractive index and temperature sensitivity of the multi-core micro/nano fiber sensor; $\Delta n$ and $\Delta T$ are the changes of the refractive index and external ambient temperature, respectively. This means that the multi-core micro/nano fiber sensor has the potential for achieving simultaneous measurement of refractive index and temperature. 


\section{Conclusions}

A multi-core micro/nano fiber sensor is developed for the requirements of high-precision measurement of refractive index. The SMF-MMF-TCF-MMF-SMF cascade scheme is proposed to form the multi-core micro/nano fiber sensor. The refractive index sensitivity of the sensor is analyzed by FEM, and the theoretical analysis shows that the sensor's refractive index sensitivity is higher than $10000 \mathrm{~nm} / \mathrm{RIU}$ if the fiber diameter is less than $4.864 \mu \mathrm{m}$. Four different sensors with $14.50 \mathrm{~mm}, 15.99 \mathrm{~mm}, 16.10 \mathrm{~mm}$, and $16.20 \mathrm{~mm}$ tapered lengths are fabricated for refractive index measurement, and experimental study shows that the SRI can reach as high as $1920.79 \mathrm{~nm} / \mathrm{RIU}$, $2665.06 \mathrm{~nm} / \mathrm{RIU}, \quad 3509.44 \mathrm{~nm} / \mathrm{RIU}, \quad$ and $5815.50 \mathrm{~nm} / \mathrm{RIU}$, respectively. When SRI reaches $5815.50 \mathrm{~nm} / \mathrm{RIU}$, the refractive index measurement error is below $0.5 \%$. The experimental results also reveal that the multi-core micro/nano fiber sensor has the advantages of stabilization and linearity. We believe that the sensor proposed in this paper can find promising applications in the fields of seawater salinity testing, biology, and chemical applications.

\section{Acknowledgment}

I want to thank everyone who helped me during the writing of this paper. I am very grateful to all the researchers in the paper, who worked hard to complete the research work of this paper. I would also like to especially thank the reviewers and editors of the journal for asking me for guidance and advice to make the paper more complete. This work was supported by the National Natural Science Foundation of China (Grant Nos. 61735011 and 61405173), the Hebei Province Natural Science Foundation of China (Grant Nos. F2016203389 and C2015003053), the Natural Science Foundation of the Xinjiang Uygur Autonomous Region (Grant No. 2018D01A25), and the Project Supported by Science and Technology Program of Hebei Province for Institutions of Higher Education (Grant No. ZD2017082).
Open Access This article is distributed under the terms of the Creative Commons Attribution 4.0 International License (http://creativecommons.org/licenses/by/4.0/), which permits unrestricted use, distribution, and reproduction in any medium, provided you give appropriate credit to the original author(s) and the source, provide a link to the Creative Commons license, and indicate if changes were made.

\section{References}

[1] Beltrán-Mejía, Felipe, Biazoli, Claudecir R, Cordeiro, and Cristiano M. B. Cordeiro, "Tapered GRIN fiber microsensor," Optics Express, 2014, 22(25): 30432-30441.

[2] K. Misiakos, I. Raptis, E. Makarona, A Botsialas, A Salapatas, P Oikonomou, et al., "All-silicon monolithic Mach-Zehnder interferometer as a refractive index and bio-chemical sensor," Optics Express, 2014, 22(22): 26803-26813.

[3] J. P. Wen, Y. A. Tang, Y. J. Zhong, and J. N. Zheng, "Measurement of refraction rate of liquids by using the least deflection angle," Laboratory Science, 2014, 17(02): 20-23.

[4] V. I. Sokolov, A. G. Savelyev, V. M. Bouznik, S. M. Igumnov, E. V. Khaydukov, S. I. Molchanova, et al., "Refractive index and dispersion of highly fluorinated acrylic monomers in the 1.5 mum telecom wavelength region measured with a spectroscopic abbe refractometer," Measurement Science and Technology, 2014, 25(7): 077001.

[5] H. B. Wu, H. Huang, M. Bai, P. B. Liu, M. Chao, J. $\mathrm{Hu}$, et al., "An ultra-low detection-limit optofluidic biosensor based on all glass Fabry-Perot cavity," Optics Express, 2014, 22(6): 31977-31983.

[6] Z. B. Liu, "Investigation and demonstration of the Mach-Zehnder Interferometer based optical fiber filter and its sensing characteristics," Ph.D. dissertation, Beijing Jiaotong University, BeiJing, 2015.

[7] F. C. Shen, C. L. Wang, Z. Y. Sun, K. M. Zhou, L. Zhang, and X. W. Shu, "Small period long period fiber grating with improved refractive index sensitivity and dual-parameter sensing ability," Optics Letters, 2017, 42(2): 199-202.

[8] C. Li, T. G. Ning, C. Zhang, J. Li, C. B. Zhang, X. D. Wen, et al., "All-fiber multipath Mach-Zehnder interferometer based on a four-core fiber for sensing applications," Sensors and Actuators A: Physical, 2016, 248: 148-154.

[9] P. C. Chen, X. W. Shu, H. Y. Cao, and K. Sugden, "Ultra-sensitive refractive index sensor based on extremely simple femtosecond-laser-induced structure," Optics Letters, 2017, 42(6): 1157-1160.

[10] C. Li, T. G. Ning, J. Li, L. Pei, C. Zhang, C. B. Zhang, et al., "Simultaneous measurement of 
refractive index, strain, and temperature based on a four-core fiber combined with a fiber Bragg grating," Optics \& Laser Technology, 2017, 90: $179-184$.

[11] H. P. Luo, "The mechanism and experimental study of high sensitivity and multi-parameters sensing based on micro/nanofiber with modes coupling," Ph.D. dissertation, Huazhong University of Science \& Technology, Wuhan, 2016.

[12] X. H. Fu, C. Q. Yang, S. W. Wang, H. Y. Xie, S. Y. Zhang, G. W. Fu, et al., "Refractive index and temperature sensor of tapered triple cladding quartz specialty fiber," Acta Optica Sinica, 2015, 35(12): 82-89.

[13] H. W. Fu, X. Yan, H. D. Li, M. Shao, N. Zhao, Q. P. Liu, et al., "Study of fiber sensor for simultaneous measurement of refractive and temperature based on a core-mismatch Mach-Zehnder interferometer," Acta Optica Sinica, 2014, 34(11): 66-71.
[14] D. R. Chen, G. F. Hu, and L. X. Chen, "Pressure/temperature sensor based on a dual-core photonic crystal fiber," in 2011 Asia Communications and Photonics Conference and Exhibition (ACP), Shanghai, China, 2011, pp. 8307.

[15] Y. Liu and W. Li, "Low-cost high-sensitivity strain and temperature sensing using graded-index multimode fibers," Applied Optics, 2007, 46(13): 2516-2519.

[16] Y. F. Lu, C. Y. Shen, C. Zhong, D. B. Chen, X. Y. Dong, and J. H. Cai, "Refractive index and temperature sensor based on double-pass $\mathrm{M}-\mathrm{Z}$ interferometer with an FBG," IEEE Photonics Technology Letters, 2014, 26(11): 1124-1127.

[17] Y. G. Liu and W. Zhang, "Temperature characteristics of micro-nanofiber bragg granting surrounded with liquids," Laser \& Optoelectronics Progress, 2017, 54(4): 125-131. 